\title{
A NOVEL INTEGRABILITY ANALYSIS OF A GENERALIZED RIEMANN TYPE HYDRODYNAMIC HIERARCHY
}

\author{
ANATOLIJ M. SAMOILENKO, YAREMA A. PRYKARPATSKYY, \\ DENIS BLACKMORE, AND ANATOLIJ K. PRYKARPATSKI
}

Received 29 May, 2017

\begin{abstract}
The complete integrability of a generalized Riemann type hydrodynamic hierarchy is studied by means of a novel combination of symplectic and differential-algebraic tools. A compatible pair of polynomial Poissonian structures, a Lax representation and a related infinite hierarchy of conservation laws are constructed. The current investigation provides an interesting glimpse of what is apparently a far wider range of applications.
\end{abstract}

2010 Mathematics Subject Classification: 17B68; 17B80; 35Q53; 35G25; 35N10; 37K35; 58J70; 58J72; 34A34; 37K05; 37K10

Keywords: Riemann type hydrodynamic hierachy, conservation laws, Lax type integrability, biHamiltonian structure, differential ideals, Lax-Noether equation, Poissonian structures

\section{INTRODUCTION}

Since the Riemann classical works on two-dimensional hydrodynamic type equations and their invariants during the last decades there has been achieved great progress $[7,8]$ in studies of their analytical properties, in particular, in stating the existence of the Poissonian representations and infinite hierarchies of conservation laws. Important results in studying the existence of hierarchies of both local and non-local conservation laws for a general type of nonlinear differential equations were obtained in $[15,16]$ by means of differential-geometric methods, which in the two-dimensional case also makes it possible to construct the corresponding Lax type representations. Recently new mathematical approaches, based on differential-algebraic and differential geometric methods and techniques, were applied in works $[5,13]$ for studying the Lax type integrability of nonlinear differential equations of Korteweg-de Vries and Riemann type. In particular, a great deal of analytical studies $[4,5,11]$ were devoted to finding the corresponding Lax-type representations of the infinite Riemann type hydrodynamical hierarchy, suggested recently (by M. Pavlov and D. Holm [9]) in the form

$$
D_{t}^{N} u=0
$$


where the differentiation $D_{t}:=\partial / \partial t+u \partial / \partial x, \quad N \in \mathbb{N}, \quad(x, t)^{\top} \in \mathbb{R}^{2}$ and $u \in C^{\infty}(\mathbb{R} / 2 \pi \mathbb{Z} ; \mathbb{R})$. It was found that the related dynamical system

$$
D_{t} u_{1}=u_{2}, \ldots, D_{t} u_{j}=u_{j+1}, \ldots, D_{t} u_{N}=0,
$$

defined on a $2 \pi$-periodic infinite-dimensional smooth functional manifold $M^{N} \subset$ $C^{\infty}\left(\mathbb{R} / 2 \pi \mathbb{Z} ; \mathbb{R}^{N}\right)$, possesses $[11,13]$ for an arbitrary integer $N \in \mathbb{N}$ a suitable Lax type representation

$$
D_{x} f=l_{N}[u ; \lambda] f, \quad D_{t} f=q_{N}(\lambda) f
$$

with $\lambda \in \mathbb{C}$ being a complex spectral parameter and $f \in L_{\infty}\left(\mathbb{R} ; \mathbb{C}^{N}\right)$ and matrices $l_{N}[u ; \lambda], q_{N}(\lambda) \in$ End $\mathbb{C}^{2}$. Here, by definition, $u_{1}:=u \in C^{\infty}\left(\mathbb{R}^{2} ; \mathbb{R}\right)$ and the differentiations

$$
D_{t}:=\partial / \partial t+u_{1} D_{x}, \quad D_{x}:=\partial / \partial x
$$

satisfy on the manifold $M^{N}$ the following commutation relationship:

$$
\left[D_{x}, D_{t}\right]=u_{1, x} D_{x} .
$$

In particular, for the cases $N=2,3$ and $N=4$ the following exact matrix polynomial in $\lambda \in \mathbb{C}$ expressions

$$
\begin{aligned}
& l_{2}[u ; \lambda]=\left(\begin{array}{cc}
\lambda u_{1, x} & u_{2, x} \\
-2 \lambda^{2} & -\lambda u_{1, x}
\end{array}\right), \quad q_{2}(\lambda)=\left(\begin{array}{cc}
0 & 0 \\
-\lambda & 0
\end{array}\right), \\
& l_{3}[u ; \lambda]=\left(\begin{array}{ccc}
\lambda^{2} u_{1, x} & -\lambda u_{2, x} & u_{3, x} \\
3 \lambda^{3} & -2 \lambda^{2} u_{1, x} & \lambda u_{3, x} \\
6 \lambda^{4} r_{3}^{(1)}[u] & -3 \lambda^{3} & \lambda^{2} u_{1, x}
\end{array}\right), q(\lambda):=\left(\begin{array}{ccc}
0 & 0 & 0 \\
\lambda & 0 & 0 \\
0 & \lambda & 0
\end{array}\right), \\
& l_{4}[u ; \lambda]=\left(\begin{array}{cccc}
-\lambda^{3} u_{1, x} & \lambda^{2} u_{2, x} & -\lambda u_{3, x} & u_{4, x} \\
-4 \lambda^{4} & 3 \lambda^{3} u_{1, x} & -2 \lambda^{2} u_{2, x} & \lambda u_{3, x} \\
-10 \lambda^{5} r_{N}^{(1)}[u] & 6 \lambda^{4} & -3 \lambda^{3} u_{1, x} & \lambda^{2} u_{2, x} \\
-20 \lambda^{6} r_{4}^{(2)}[u] & 10 \lambda^{5} r_{4}^{(1)}[u] & -4 \lambda^{4} & \lambda^{3} u_{1, x}
\end{array}\right), \\
& q_{4}(\lambda):=\left(\begin{array}{cccc}
0 & 0 & 0 \\
\lambda & 0 & 0 & 0 \\
0 & \lambda & 0 & 0 \\
0 & 0 & \lambda & 0
\end{array}\right),
\end{aligned}
$$

were presented in exact form.

In the present work we will be interseted in studying the complete integrability of a new important dispersionless Riemann type hydrodynamic hierarchy

$$
D_{t}^{N-1} u=\bar{z}_{x}^{s}, \quad D_{t} \bar{z}=0
$$


on a $2 \pi$-periodic functional manifold $\bar{M}^{N} \subset C^{\infty}\left(\mathbb{R} / 2 \pi \mathbb{Z} ; \mathbb{R}^{N}\right)$, where $s, N \in \mathbb{N}$ are arbitrary natural numbers, the vector $\left(u, D_{t} u, D_{t}^{2} u, \ldots, D_{t}^{N-1} u, \bar{z}\right)^{\top} \in \bar{M}^{N}$, the differentiations $D_{x}:=\partial / \partial x, D_{t}:=\partial / \partial t+u \partial / \partial x$ satisfy as above the Lie-algebraic commutator relationship (1.5) and $t \in \mathbb{R}$ is an evolution parameter. This system can be considered at $s=2$ and $N=3$ as a nontrivial generalization of the dispersionless Riemann hydrodynamic system (1.1), extensively studied by means of different mathematical tools in $[4,5,10,11,13,14]$. For the case $s=2$ and $N=2$ it is well known $[1,12]$ that the system (1.7) is a smooth Lax integrable bi-Hamiltonian flow on the $2 \pi$-periodic functional manifold $\bar{M}_{2}$, whose Lax representation is given by the compatible linear system

$$
D_{x} f=\left(\begin{array}{cc}
\bar{z}_{x} & 0 \\
-\lambda\left(u+u_{x} / \bar{z}_{x}\right) & -\bar{z}_{x x} / \bar{z}_{x}
\end{array}\right) f, \quad D_{t} f=\left(\begin{array}{cc}
0 & 0 \\
-\lambda \bar{z}_{x} & \left.u_{x}\right)
\end{array}\right) f,
$$

where $f \in C^{\infty}\left(\mathbb{R}^{2} ; \mathbb{R}^{2}\right)$ and $\lambda \in \mathbb{R}$ is an arbitrary spectral parameter.

At $s=2$ and $N=3$ dynamical system (1.7) is equivalent to that on a $2 \pi$-periodic functional manifold $\bar{M}_{3} \subset C^{\infty}\left(\mathbb{R} / 2 \pi \mathbb{Z} ; \mathbb{R}^{3}\right)$ for a vector $(u, v, \bar{z})^{\top} \in \bar{M}_{3}$ :

$$
D_{t} u=v, \quad D_{t} v=\bar{z}_{x}^{2}, \quad D_{t} \bar{z}=0 .
$$

The latter can be easily rewritten by means of the change of variables $z:=\bar{z}_{x}^{2}$ as that on a $2 \pi$-periodic functional manifold $M_{3} \subset C^{\infty}\left(\mathbb{R} / 2 \pi \mathbb{Z} ; \mathbb{R}^{3}\right)$ for a vector $(u, v, z)^{\top} \in M_{3}$

$$
D_{t} u=v, \quad D_{t} v=z, \quad D_{t} z=-2 z u_{x},
$$

or in the form of the flow

$$
\left(\begin{array}{l}
d u / d t \\
d v / d t \\
d z / d t
\end{array}\right)=K[u, v, z]:=\left(\begin{array}{c}
v-u u_{x} \\
z-u v_{x} \\
-2 u_{x} z-u z_{x}
\end{array}\right),
$$

defining a standard smooth dynamical system on the infinite-dimensional functional manifold $M_{3}$, where $K: M_{3} \rightarrow T\left(M_{3}\right)$ is the corresponding vector field on $M_{3}$.

Concerning the new dynamical system (1.11) we succeeded in proving the following result based on the symplectic gradient-holonomic and differential algebraic tools.

Proposition 1. The Riemann type hydrodynamic flow (1.11) is a bi-Hamiltonian dynamical system on the functional manifold $M_{3}$ with respect to two compatible Poissonian structures $\vartheta, \eta: T^{*}\left(M_{3}\right) \rightarrow T\left(M_{3}\right)$

$\vartheta:=\left(\begin{array}{ccc}0 & 1 & 0 \\ -1 & 0 & 0 \\ 0 & 0 & 2 z^{1 / 2} D_{x} z^{1 / 2}\end{array}\right), \eta:=\left(\begin{array}{ccc}\partial^{-1} & u_{x} \partial^{-1} & 0 \\ \partial^{-1} u_{x} & v_{x} \partial^{-1}+\partial^{-1} v_{x} & \partial^{-1} z_{x}-2 z \\ 0 & z_{x} \partial^{-1}+2 z & 0\end{array}\right)$, 
possessing an infinite hierarchy of mutually commuting conservation laws and a nonautonomous Lax representation of the form

$$
\begin{aligned}
D_{t} f & =\left(\begin{array}{ccc}
0 & 0 & 0 \\
-\lambda & 0 & 0 \\
0 & -\lambda \sqrt{z} & u_{x}
\end{array}\right) f, \\
D_{x} f & =\left(\begin{array}{ccc}
\lambda^{2} u \sqrt{z} & \lambda v \sqrt{z} & z \\
-\lambda^{3} t u \sqrt{z} & -\lambda^{2} t v \sqrt{z} & -\lambda t z \\
\lambda^{4}\left(t u v-u^{2}\right)- & -\lambda v_{x} / \sqrt{z}+ & -\lambda^{2} \sqrt{z}(u-t v)- \\
-\lambda^{2} u_{x} / \sqrt{z} & +\lambda^{3}\left(t v^{2}-u v\right) & -z_{x} / 2 z
\end{array}\right) f,
\end{aligned}
$$

where $\lambda \in \mathbb{R}$ is an arbitrary spectral parameter and $f \in C^{\infty}\left(\mathbb{R}^{2} ; \mathbb{R}^{3}\right)$.

\section{SYMPLECTIC GRADIENT-HOLONOMIC INTEGRABILITY ANALYSIS}

Our first steps in proving Proposition 1 are fashioned using the symplectic gradientholonomic method, which takes us a long way towards the desired result.

\subsection{Poissonian structure analysis on the functional manifold $M_{3}$}

By employing the symplectic gradient-holonomic approach $[1,6,12]$ to studying the integrability of smooth nonlinear dynamical systems on functional manifolds, one can find a set of conservation laws for (1.11) by constructing solutions $\varphi:=$ $\varphi[u, v, z] \in T^{*}\left(M_{3}\right)$ to the functional Lax gradient equation:

$$
d \varphi / d t+K^{\prime, *} \varphi=\operatorname{grad} \mathscr{L},
$$

where $\varphi^{\prime}=\varphi^{\prime, *}, \mathscr{L} \in \mathscr{D}\left(M_{3}\right)$ is a suitable Lagrangian functional (in the space of smooth functionals on $\left.M_{3}\right)$ and the linear operator $K^{\prime, *}: T^{*}\left(M_{3}\right) \rightarrow T^{*}\left(M_{3}\right)$ is the adjoint with respect to the standard convolution $(\cdot, \cdot)$ on $T^{*}\left(M_{3}\right) \times T\left(M_{3}\right)$, of the Fréchet-derivative of a nonlinear mapping $K: M_{3} \rightarrow T\left(M_{3}\right)$; namely,

$$
K^{\prime, *}=\left(\begin{array}{ccc}
u D_{x} & -v_{x} & z_{x}+2 z D_{x} \\
1 & u_{x}+u D_{x} & 0 \\
0 & 1 & -u_{x}+u D_{x}
\end{array}\right) .
$$

The Lax gradient equation (2.1) can be, owing to (1.11), rewritten as

$$
D_{t} \varphi+k[u, v, z] \varphi=\operatorname{grad} \mathscr{L},
$$

where the matrix operator

$$
k[u, v, z]:=\left(\begin{array}{ccc}
0 & -v_{x} & z_{x}+2 z D_{x} \\
1 & u_{x} & 0 \\
0 & 1 & -u_{x}
\end{array}\right) .
$$

The first vector elements

$$
\varphi_{\vartheta}[u, v, z]=\left(z-u v_{x},-v+u u_{x}, u\right), \mathscr{L}_{\vartheta}=0
$$




$$
\begin{aligned}
& \varphi_{\eta}[u, v, z]=\left(v_{x},-u_{x},-1\right)^{\top}, \mathscr{L}_{\eta}=0, \\
& \varphi_{0}[u, v, z]=\left(-\left(u_{x} z^{-1 / 2}\right)_{x},\left(z^{-1 / 2}\right)_{x},\left(v_{x} / 2-u_{x}^{2} / 4\right) z^{-3 / 2}\right)^{\top}, \mathscr{L}_{0}=0,
\end{aligned}
$$

as can be easily checked, are solutions of the functional equation (2.3). From an application of the standard Volterra homotopy formula

$$
H:=\int_{0}^{1} d \mu\left(\varphi[\mu u, \mu v, \mu z],(u, v, z)^{\top}\right),
$$

one finds the conservation laws for (1.11); namely,

$$
\begin{aligned}
& H_{\eta}=\frac{1}{2} \int_{0}^{2 \pi} d x\left(2 u z-v^{2}-u^{2} v_{x}\right) \\
& H_{\vartheta}:=\int_{0}^{2 \pi} d x\left(u v_{x} / 2-v u_{x} / 2-z\right), \quad H_{0}:=\frac{1}{2} \int_{0}^{2 \pi} d x\left(u_{x}^{2}-2 v_{x}\right) z^{-1 / 2} .
\end{aligned}
$$

It is now quite easy, making use of the conservation laws (2.7), to construct a Poissonian structure $\vartheta: T^{*}\left(M_{3}\right) \rightarrow T\left(M_{3}\right)$ for the dynamical system (1.11). If we use the representations

$$
\begin{aligned}
& H_{\vartheta}=\int_{0}^{2 \pi} d x\left(u v_{x} / 2-v u_{x} / 2-z\right):=\left(\psi_{\vartheta},\left(u_{x}, v_{x}, z_{x}\right)^{\top}\right), \\
& \psi_{\vartheta}:=\left(-v / 2, u / 2, z^{-1 / 2} D_{x}^{-1} z^{1 / 2} / 2\right)^{\top},
\end{aligned}
$$

it follows that the vector $\psi_{\vartheta} \in T^{*}\left(M_{3}\right)$ satisfies the Lax gradient equation (2.3):

$$
D_{t} \psi_{\vartheta}+k[u, v, z] \psi_{\vartheta}=\operatorname{grad} \mathscr{L}_{\vartheta}
$$

where the Lagrangian function $\mathscr{L}_{\vartheta}=\left(\psi_{\vartheta}, K\right)-H_{\vartheta}$. Thus, based on the inverse cosymplectic functional expression

$$
\vartheta^{-1}:=\psi_{\vartheta}^{\prime}-\psi_{\vartheta}^{\prime, *}=\left(\begin{array}{ccc}
0 & -1 & 0 \\
1 & 0 & 0 \\
0 & 0 & z^{-1 / 2} D_{x}^{-1} z^{-1 / 2} / 2
\end{array}\right)
$$

one readily obtains the linear co-symplectic operator on the manifold $M_{3}$ :

$$
\vartheta:=\left(\begin{array}{ccc}
0 & 1 & 0 \\
-1 & 0 & 0 \\
0 & 0 & 2 z^{1 / 2} D_{x} z^{1 / 2}
\end{array}\right)
$$

which is the corresponding Poissonian operator for the dynamical system (1.11). It is also important to observe that the dynamical system (1.11) is a Hamiltonian flow on the functional manifold $M_{3}$ with respect to the Poissonian structure (2.11).

$$
K[u, v, z]=-\vartheta \operatorname{grad} H_{\eta}
$$




\subsection{Poissonian structure analysis on $\bar{M}_{3}$}

In what follows, we shall find it convenient to construct other Poissonian structures for dynamical system (1.9) on the manifold $\bar{M}_{3}$, rewritten in the equivalent form

$$
\frac{d}{d t}\left(\begin{array}{c}
u \\
v \\
\bar{z}
\end{array}\right)=\bar{K}[u, v, \bar{z}]:=\left(\begin{array}{c}
v-u u_{x} \\
\bar{z}_{x}^{2}-u v_{x} \\
0
\end{array}\right),
$$

where $\bar{K}: \bar{M}_{3} \rightarrow T\left(\bar{M}_{3}\right)$ is the corresponding vector field on $\bar{M}_{3}$. To proceed, we need to obtain additional solutions to the Lax gradient equation (2.3) on the functional manifold $\bar{M}_{3}$

$$
D_{t} \bar{\psi}+\bar{k}[u, v, z] \bar{\psi}=\operatorname{grad} \overline{\mathscr{L}},
$$

where the matrix operator is

$$
\bar{k}[u, v, \bar{z}]:=\left(\begin{array}{ccc}
0 & -v_{x} & -\bar{z}_{x} \\
1 & u_{x} & 0 \\
0 & -2 \partial \bar{z}_{x} & u_{x}
\end{array}\right),
$$

and which we may rewrite in the componentwise form

$$
\begin{aligned}
& D_{t} \bar{\psi}^{(1)}=v_{x} \bar{\psi}^{(2)}+\bar{z}_{x} \bar{\psi}^{(3)}+\delta \overline{\mathscr{L}} / \delta u, \\
& D_{t} \bar{\psi}^{(2)}=-\bar{\psi}^{(1)}-u_{x} \bar{\psi}^{(2)}+\delta \overline{\mathscr{L}} / \delta v, \\
& D_{t} \bar{\psi}^{(3)}=2\left(\bar{z}_{x} \bar{\psi}^{(2)}\right)_{x}-u_{x} \bar{\psi}^{(3)}+\delta \overline{\mathscr{L}} / \delta \bar{z},
\end{aligned}
$$

where the vector $\bar{\psi}:=\left(\bar{\psi}^{(1)}, \bar{\psi}^{(2)}, \bar{\psi}^{(3)}\right)^{\top} \in T^{*}\left(\bar{M}_{3}\right)$. As a simple consequence of (2.16), one obtains the following system of differential relationships:

$$
\begin{gathered}
D_{t}^{3} \tilde{\psi}^{(2)}=-2 \bar{z}_{x}^{2} \tilde{\psi}_{x}^{(2)}+D_{t}^{2} \partial^{-1}(\delta \overline{\mathscr{L}} / \delta v)- \\
-\partial^{-1}<\operatorname{grad} \overline{\mathscr{L}}_{,}\left(u_{x}, v_{x}, \bar{z}_{x}\right)^{\top}>, \\
D_{t} \tilde{\psi}^{(2)}=-\tilde{\psi}^{(1)}+\partial^{-1}(\delta \overline{\mathscr{L}} / \delta v), \\
D_{t} \tilde{\psi}^{(3)}=2 \bar{z}_{x} \tilde{\psi}_{x}^{(2)}+\partial^{-1}(\delta \overline{\mathscr{L}} / \delta \bar{z}) .
\end{gathered}
$$

Here we have defined $\left(\bar{\psi}^{(1)}, \bar{\psi}^{(2)}, \bar{\psi}^{(3)}\right)^{\top}:=\left(\tilde{\psi}_{x}^{(1)}, \tilde{\psi}_{x}^{(2)}, \tilde{\psi}_{x}^{(3)}\right)^{\top}$ and made use of the commutator relationship for differentiations $D_{t}$ and $D_{x}$ :

$$
\left[D_{t}, \alpha^{-1} D_{x}\right]=0 \text {, }
$$

which holds for the function $\alpha:=1 / \bar{z}_{x}$, where $D_{t} \bar{z}=0$. It therefore follows that after solving the first equation of system (2.17), one can recursively sole the remaining two equations. In particular, it is easy to see that the three vector elements

$$
\begin{gathered}
\tilde{\psi}_{0}=\left(-v, u,-2 \bar{z}_{x}\right)^{\top}, \overline{\mathscr{L}}_{0}=0 ; \\
\tilde{\psi}_{\theta}=\left(-u_{x} / \bar{z}_{x}, 1 / \bar{z}_{x},\left(u_{x}^{2}-2 v_{x}\right) /\left(2 \bar{z}_{x}^{2}\right)\right)^{\top}, \overline{\mathscr{L}}_{\theta}=0 ; \\
\tilde{\psi}_{\eta}=\left(u / 2,-x / 2, \partial^{-1}\left[\left(2 v_{x}-u_{x}^{2}\right) /\left(2 \bar{z}_{x}\right)\right]\right), \overline{\mathscr{L}}_{\eta}=\left(D_{x} \tilde{\psi}_{\eta}, \bar{K}\right)-H_{\vartheta},
\end{gathered}
$$


are solutions of the system (2.17). The first two elements of (2.19) lead to the Volterra symmetric vectors $\bar{\psi}_{0}=D_{x} \tilde{\psi}_{0}, \bar{\psi}_{\theta}=D_{x} \tilde{\psi}_{\theta} \in T^{*}\left(\bar{M}_{3}\right): \bar{\psi}_{0}^{\prime}=\bar{\psi}_{0}^{\prime \prime *}, \bar{\psi}_{\theta}^{\prime}=\bar{\psi}_{\theta}^{\prime, *}$ entailing the trivial conservation laws $\left(\bar{\psi}_{0}, \bar{K}\right)=0=\left(\bar{\psi}_{\theta}, \bar{K}\right)$. The third element of (2.19) gives rise to the Volterra asymmetric vector $\bar{\psi}_{\eta}:=D_{x} \tilde{\psi}_{\eta}: \bar{\psi}_{\eta}^{\prime} \neq \bar{\psi}_{\eta}^{\prime, *}$, entailing the following inverse co-symplectic functional expression:

$$
\bar{\eta}^{-1}:=\bar{\psi}_{\eta}^{\prime}-\bar{\psi}_{\eta}^{\prime, *}=\left(\begin{array}{ccc}
\partial & 0 & -\partial \frac{u_{x}}{\bar{z}_{x}} \\
0 & 0 & \partial \frac{\overline{\bar{z}}_{x}}{\bar{z}_{x}} \\
-\frac{u_{x}}{\bar{z}_{x}} \partial & \frac{1}{\bar{z}_{x}} \partial & -\frac{v_{x}}{\bar{z}_{x}} \partial \frac{1}{\bar{z}_{x}} \partial \frac{u_{x}}{\bar{z}_{x}}-\frac{1}{\bar{z}_{x}} \partial \partial_{\bar{z}_{x}}
\end{array}\right) .
$$

Correspondingly, the Poissonian operator $\bar{\eta}: T^{*}\left(\bar{M}_{3}\right) \rightarrow T\left(\bar{M}_{3}\right)$ is

$$
\bar{\eta}=\left(\begin{array}{ccc}
\partial^{-1} & u_{x} \partial^{-1} & 0 \\
\partial^{-1} u_{x} & v_{x} \partial^{-1}+\partial^{-1} v_{x} & \partial^{-1} \bar{z}_{x} \\
0 & \bar{z}_{x} \partial^{-1} & 0
\end{array}\right),
$$

subject to which the following Hamiltonian representation

$$
\bar{K}[u, v, \bar{z}]=-\left.\bar{\eta} \operatorname{grad} H_{\eta}\right|_{z=\bar{z}_{x}^{2}}
$$

holds on the manifold $\bar{M}_{3}$.

\subsection{Hamiltonian integrability analysis}

Next, we return to our integrability analysis of the dynamical system (1.11) on the functional manifold $M_{3}$. It is easy to recalculate the form of the Poissonian operator (2.21) on the manifold $\bar{M}_{3}$ to that acting on the manifold $M_{3}$, giving rise to the second Hamiltonian representation of (1.11):

$$
K[u, v, z]=-\eta \operatorname{grad} H_{\vartheta},
$$

where $\eta: T^{*}\left(M_{3}\right) \rightarrow T\left(M_{3}\right)$ is the corresponding Poissonian operator. As a next important point, the Poissonian operators $(2.11)$ and (2.21) are compatible [1-3,12] on the manifold $\bar{M}_{3}$; that is, the operator pencil $(\vartheta+\lambda \eta): T^{*}\left(M_{3}\right) \rightarrow T\left(M_{3}\right)$ is also Poissonian for arbitrary $\lambda \in \mathbb{R}$. As a consequence, any operator of the form

$$
\vartheta_{n}:=\vartheta\left(\vartheta^{-1} \eta\right)^{n}
$$

for all $n \in \mathbb{Z}$ is Poissonian on the manifold $M_{3}$. Using now the homotopy formula (2.6) and recursion property of the Poissonian pair (2.12) and (2.21), it is easy to construct the related infinite hierarchy of mutually commuting conservation laws

$$
\begin{gathered}
\gamma_{j}=\int_{0}^{1} d \mu\left(\operatorname{grad} \gamma_{j}[\mu u, \mu v, \mu z],(u, v, z)^{\top}\right), \\
\operatorname{grad} \gamma_{j}[u, v, z]:=\Lambda^{j} \operatorname{grad} H_{\eta},
\end{gathered}
$$


for the dynamical system (1.11), where $j \in \mathbb{Z}_{+}$and $\Lambda:=\vartheta^{-1} \eta: T^{*}\left(M_{3}\right) \rightarrow$ $T^{*}\left(M_{3}\right)$ is the corresponding recursion operator, which satisfies the so called associated Lax commutator relationship

$$
d \Lambda / d t=\left[\Lambda, K^{\prime, *}\right] .
$$

In the course of above analysis and observations, we have proved the following result.

Proposition 2. The Riemann hydrodynamic system (1.11) is a bi-Hamiltonian dynamical system on the functional manifold $M_{3}$ with respect to the compatible Poissonian structures $\vartheta, \eta: T^{*}\left(M_{3}\right) \rightarrow T\left(M_{3}\right)$

$\vartheta:=\left(\begin{array}{ccc}0 & 1 & 0 \\ -1 & 0 & 0 \\ 0 & 0 & 2 z^{1 / 2} D_{x} z^{1 / 2}\end{array}\right), \eta:=\left(\begin{array}{ccc}\partial^{-1} & u_{x} \partial^{-1} & 0 \\ \partial^{-1} u_{x} & v_{x} \partial^{-1}+\partial^{-1} v_{x} & \partial^{-1} z_{x}-2 z \\ 0 & z_{x} \partial^{-1}+2 z & 0\end{array}\right)$

and possesses an infinite hierarchy of mutually commuting conservation laws (2.25).

Concerning the existence of an additional infinite and parametrically $\mathbb{R} \ni \lambda$-ordered hierarchy of conservation laws for the dynamical system (1.11), it is instructive to consider the dispersive nonlinear dynamical system

$$
\left(\begin{array}{c}
d u / d \tau \\
d v / d \tau \\
d z / d \tau
\end{array}\right)=-\vartheta \operatorname{grad} H_{0}[u, v, z]:=\left(\begin{array}{c}
-\left(z^{-1 / 2}\right)_{x} \\
-\left(u_{x} z^{-1 / 2}\right)_{x} \\
z^{1 / 2}\left(\frac{u_{x}^{2}-2 v_{x}}{2 z}\right)_{x}
\end{array}\right)=\tilde{K}[u, v, z] .
$$

By solving the corresponding Lax equation

$$
d \tilde{\varphi} / d t+\tilde{K}^{\prime, *} \tilde{\varphi}=0
$$

for an element $\tilde{\varphi} \in T^{*}\left(M_{3}\right)$ in a suitably chosen asymptotic form, one can construct an infinite ordered hierarchy of conservation laws for (1.11), which we will not delve into here. This hierarchy and the existence of an infinite and parametrically $\mathbb{R} \ni \lambda$ ordered hierarchy of conservation laws for the Riemann type dynamical system (1.11) provided compelling indications that it is completely integrable in the sense of Lax on the functional manifold $M_{3}$. We shall complete our integrability analysis in the next section using rather powerful differential-algebraic tools that were devised recently in $[11,13,14]$.

\section{Differential-ALGEBRAIC INTEGRABILITy ANALYSis: $N=3$}

Consider a polynomial differential ring $\mathcal{K}\{u\} \subset \mathcal{K}:=\mathbb{R}\{\{x, t\}\}$ generated by a fixed functional variable $u \in \mathbb{R}\{\{x, t\}\}$ and invariant with respect to two differentiations $D_{x}:=\partial / \partial x$ and $D_{t}:=\partial / \partial t+u \partial / \partial x$ that satisfy the Lie-algebraic commutator relationship (1.5)

$$
\left[D_{x}, D_{t}\right]=u_{x} D_{x}
$$


together with the constraint (1.10) expressed in the differential-algebraic functional form

$$
D_{t}^{3} u=-2 D_{t}^{2} u D_{x} u \text {. }
$$

Since the Lax representation for the dynamical system (1.11) can be interpreted [1, 13] as the existence of a finite-dimensional invariant ideal $\mathcal{L}\{u\} \subset \mathcal{K}\{u\}$ realizing the corresponding finite-dimensional representation of the the Lie-algebraic commutator relationship (3.1), this ideal can be constructed as

$$
\mathcal{L}\{u\}:=\left\{\lambda^{2} u f_{1}+\lambda v f_{2}+z^{1 / 2} f_{3} \in \mathcal{K}\{u\}: f_{j} \in \mathcal{K}, 1 \leq j \leq 3, \lambda \in \mathbb{R}\right\},
$$

where $v=D_{t} u, z=D_{t}^{2} u$ and $\lambda \in \mathbb{R}$ is an arbitrary real parameter. To find finitedimensional representations of the $D_{x}$ - and $D_{t}$-differentiations, it is necessary [13] first to find the $D_{t}$-invariant kernel ker $D_{t} \subset \mathcal{d}\{u\}$ and next to check its invariance with respect to the $D_{x}$-differentiation. It is easy to show that

$$
\operatorname{ker} D_{t}=\left\{f \in \mathcal{K}^{3}\{u\}: D_{t} f=q(\lambda) f, \quad \lambda \in \mathbb{R}\right\},
$$

where the matrix $q(\lambda):=q[u, v, z ; \lambda] \in$ End $\mathcal{K}\{u\}^{3}$ is given as

$$
q(\lambda)=\left(\begin{array}{ccc}
0 & 0 & 0 \\
-\lambda & 0 & 0 \\
0 & -\lambda \sqrt{z} & u_{x}
\end{array}\right) .
$$

To obtain the corresponding representation of the $D_{x}$-differentiation in the space $\mathcal{K}^{3}$, it suffices to find a matrix $l(\lambda):=l[u, v, z ; \lambda] \in$ End $\mathcal{K}\{u\}^{3}$ such that

$$
D_{x} f=l(\lambda) f
$$

for $f \in \mathcal{K}\{u\}^{3}$ and the related ideal

$$
\mathcal{R}\{u\}:=\left\{<g, f>_{\mathcal{K}^{3}}: f \in \operatorname{ker} D_{t} \subset \mathcal{K}^{3}\{u\}, g \in \mathcal{K}^{3}\right\}
$$

is $D_{x}$-invariant with respect to the matrix differentiation representation (3.5). Straightforward calculations using this invariance condition then yield the following matrix

$$
l(\lambda)=\left(\begin{array}{ccc}
\lambda^{2} u \sqrt{z} & \lambda v \sqrt{z} & z \\
-\lambda^{3} t u \sqrt{z} & -\lambda^{2} t v \sqrt{z} & -\lambda t z \\
\lambda^{4}\left(t u v-u^{2}\right)- & -\lambda v_{x} / \sqrt{z}+ & -\lambda^{2} \sqrt{z}(u-t v)- \\
-\lambda^{2} u_{x} / \sqrt{z} & +\lambda^{3}\left(t v^{2}-u v\right) & -z_{x} / 2 z
\end{array}\right)
$$

entering the linear equation (3.5). Thus, the following proposition is proved.

Proposition 3. The generalized Riemann type dynamical system (1.11) is a biHamiltonian integrable flow possessing a non-autonomous Lax representation of the form

$$
D_{t} f=\left(\begin{array}{ccc}
0 & 0 & 0 \\
-\lambda & 0 & 0 \\
0 & -\lambda \sqrt{z} & u_{x}
\end{array}\right) f
$$




$$
D_{x} f=\left(\begin{array}{ccc}
\lambda^{2} u \sqrt{z} & \lambda v \sqrt{z} & z \\
-\lambda^{3} t u \sqrt{z} & -\lambda^{2} t v \sqrt{z} & -\lambda t z \\
\lambda^{4}\left(t u v-u^{2}\right)- & -\lambda v_{x} / \sqrt{z}+ & -\lambda^{2} \sqrt{z}(u-t v)- \\
-\lambda^{2} u_{x} / \sqrt{z} & +\lambda^{3}\left(t v^{2}-u v\right) & -z_{x} / 2 z
\end{array}\right) f
$$

where $\lambda \in \mathbb{R}$ is an arbitrary spectral parameter and $f \in C^{\infty}\left(\mathbb{R}^{2} ; \mathbb{R}^{3}\right)$.

Remark 1. Simple analogs of the above differential-algebraic calculations for the case $N=2$ lead readily to the corresponding Riemann type hydrodynamic system

$$
D_{t} u=\bar{z}_{x}^{2}, \quad D_{t} \bar{z}=0
$$

on the functional manifold $\bar{M}_{2}$, which possesses the following matrix Lax representation:

$$
D_{t} f=\left(\begin{array}{cc}
0 & 0 \\
-\lambda \bar{z}_{x} & u_{x}
\end{array}\right), \quad D_{x} f=\left(\begin{array}{cc}
\bar{z}_{x} & 0 \\
-\lambda\left(u+u_{x} / \bar{z}_{x}\right. & -\bar{z}_{x x} / \bar{z}_{x}
\end{array}\right) f
$$

where $\lambda \in \mathbb{R}$ is an arbitrary spectral parameter and $f \in C^{\infty}\left(\mathbb{R}^{2} ; \mathbb{R}^{2}\right)$.

As one can readily see, these differential-algebraic results provide a direct proof of Proposition 1 describing the integrability of system (1.11) for $N=3$. The matrices (3.7) are not of standard form since they depend explicitly on the temporal evolution parameter $t \in \mathbb{R}$. Nonetheless, the matrices (3.4) and (3.7) satisfy for all $\lambda \in \mathbb{R}$ the well-known Zakharov-Shabat type compatibility condition

$$
D_{t} l(\lambda)=[q(\lambda), l(\lambda)]+D_{x} l(\lambda)-u_{x} l(\lambda),
$$

which follows from the Lax type relationships (3.3) and (3.5)

$$
D_{t} f=q(\lambda) f, \quad D_{x} f=l(\lambda) f
$$

and the commutator condition (3.1). Moreover, taking into account that the dynamical system (1.11) has a compatible Poissonian pair (2.11) and (2.21) depending only on the variables $(u, v, z)^{\top} \in M_{3}$ and not depending on the temporal variable $t \in \mathbb{R}$, one can certainly assume that it also possesses a standard autonomous Lax representation, which can possibly be found by means of a suitable gauge transformation of (3.12). We plan to pursue this line of analysis in a forthcoming paper.

\section{CONCLUding REMARKS}

A new nonlinear Hamiltonian dynamical system representing a Riemann type hydrodynamic equation (1.7) in two and three dimensions proves to be a very interesting example of a Lax integrable dynamical system, as we have proved here. In particular, the integrability prerequisites of this dynamical system, such as compatible Poissonian structures, an infinite hierarchy of conservation laws and related Lax representation have been constructed by means of both the symplectic gradient-holonomic approach $[1,6,12]$ and innovative differential-algebraic tools devised recently $[4,13]$ 
for analyzing the integrability of a special infinite hierarchy of Riemann type hydrodynamic systems. It is also quite clear from recent research in this area and our work in this paper that the dynamical system (1.7) is a Lax integrable bi-Hamiltonian flow for arbitrary integers $N \in \mathbb{N}$; this is perhaps most readily verified by means of the differential-algebraic approach, which was devised and successfully applied here for the cases $N=2$ and 3 .

We have seen in the course of this investigation that perhaps the most important lesson that one can derive from this approach is the following: If an analysis of a given nonlinear Hamiltonian dynamical system via the gradient-holonomic method indicates (but does not necessarily prove) that the system is Lax integrable, then its Lax representation can often be shown to exist and then successfully derived by means of a suitably constructed invariant differential ideal $\mathcal{L}\{u\}$ of the ring $\mathcal{K}\{u\}$ in accordance with the differential-algebraic approach developed here. Consequently, when it comes to applying this lesson to the investigation of other nonlinear dynamical systems, it is natural to start with systems that are known to be Lax integrable and then to try to identify and characterize those algebraic structures responsible for the existence of a related finite-dimensional matrix representation for the basic $D_{x}$ and $D_{t}$-differentiations in a vector space $\mathcal{K}^{p}$ for some finite $p \in \mathbb{Z}_{+}$.

It seems plausible that if one could do this for several classes of Lax integrable dynamical systems, certain patterns in the algebraic structures may be detected that can be used to assemble a more extensive array of symplectic and differential-algebraic tools capable of resolving the question of complete integrability for many other types of nonlinear Hamiltonian dynamical systems. Moreover, if the integrability is established in this manner, the approach should also serve as a means of constructing associated artifacts of the integrability such as Lax representations and hierarchies of mutually commuting invariants. As a particular differential-algebraic problem of interest concerning these matrix representations, one can seek to develop a scheme for the effective construction of functional generators of the corresponding invariant finite-dimensional ideals $d\{u\} \subset \mathcal{K}\{u\}$ under given differential-algebraic constraints imposed on the $D_{x}$ - and $D_{t}$-differentiations.

We have demonstrated here that an approach combining the gradient-holonomic method with some recently devised differential-algebraic techniques can be a very effective and efficient way of investigating integrability for a particular class of infinitedimensional Hamiltonian dynamical systems (generalized Riemann hydrodynamical systems). But a closer look at the specific details of the approach reveals, we believe, that this combination of methods can be adapted to perform effective integrability investigations of a much wider range of dynamical systems - a goal that we intend to pursue in the near future. 


\section{ACKNOWLEDGEMENTS}

Authors are much obliged to Profs. J. Cieśliński, M. Błaszak and M.Pavlov for very instrumental discussion of the work, valuable advice, comments and remarks. Special thanks are due the Scientific and Technological Research Council of Turkey (TUBITAK) for partial support of the research by A.K. Prykarpatski, and the National Science Foundation (Grant CMMI-1029809) for partial support of the research of D. Blackmore.

\section{REFERENCES}

[1] D. Blackmore, A. Prykarpatsky, and V. Samoylenko, Nonlinear dynamical systems of mathematical physics: spectral and differential-geometrical integrability analysis. New Jersey: World Scientific, 2011. doi: 13/978-981-4327-15-2.

[2] D. Błaszak, Multi-Hamiltonian theory of dynamical systems. Berlin: Springer, $1998 . \quad$ doi: 13/978-3642637803.

[3] L. Faddeev and L. Takhtadjan, Hamiltonian methods in the theory of solitons. Berlin: Springer, 2007. doi: 13/978-3-540-69843-2.

[4] J. Golenia, N. Bogolubov (jr.), Z. Popowicz, M. Pavlov, and A. Prykarpatsky, "A new Riemann type hydrodynamical hierarchy and its integrability analysis." http://publications.ictp.it Preprint ICTP - IC/2009/0959, 2009, 2009.

[5] J. Golenia, M. Pavlov, Z. Popowicz, , and A. Prykarpatsky, "On a nonlocal Ostrovsky-Whitham type dynamical system, its Riemann type inhomogenious regularizations and their integrability." SIGMA, vol. 62, no. 1, pp. 1-20, 1985, doi: 10.3842/SIGMA.2010.002.

[6] O. Hentosh, M. Prytula., and A. Prykarpatsky, Differential-geometric and Lie-algebraic foundations of investigating nonlinear dynamical systems on functional manifolds. The Second edition. Lviv: Lviv National University, 2006. doi: 13/966-613-394-6.

[7] M.Pavlov, "Hamiltonian formalism of weakly nonlinear hydrodynamic systems." Theor. Math. Phys., vol. 73, no. 2, pp. 316-320, 1987, doi: 10.1007/BF01034820.

[8] S. P. Novikov, "The geometry of conservative systems of hydrodynamic type. The method of averaging for field-theoretical systems," Russ. Math. Surv., vol. 40, no. 4, pp. 85-98, 1985.

[9] M. Pavlov and D. Holm, "Private communication."

[10] Z. Popowicz, "The matrix Lax representation of the generalized Riemann equations and its conservation laws." Phys. Lett. A, vol. 375, no. 7, pp. 3268-3272, 2011, doi: 10.1016/j.physleta.2011.06.068.

[11] Z. Popowicz and A. Prykarpatsky, "The non-polynomial conservation laws and integrability analysis of generalized Riemann type hydrodynamical equations," Nonlinearity, vol. 23, no. 10, pp. 2517-2537, 2010, doi: 10.1088/0951-7715/23/10/010.

[12] A. Prykarpatsky and I. Mykytyuk, Algebraic integrability of nonlinear dynamical systems on manifolds: classical and quantum aspects. Dordrecht/Boston: Kluwer Publisher, 1998. doi: 10.1007/978-94-011-4994-5.

[13] A. Prykarpatsky, O. Artemovych, Z. Popowicz, and M. Pavlov, "Differential-algebraic integrability analysis of the generalized Riemann type and Korteweg-de Vries hydrodynamical equations.” J. Phys. A: Math. Theor. , vol. 43, no. 7, pp. 295 205-295 218, 2010, doi: 10.1088/17518113/43/29/295205.

[14] A. Prykarpatsky, O. Artemovych, Z. Popowicz, and M. Pavlov, "The differential-algebraic and bi-Hamiltonian integrability analysis of the Riemann type hierarchy revisited." J. Math. Phys. , vol. 53, no. 10, pp. 103 521-103 534, 2012, doi: 10.1063/1.4761821. 
[15] V. Vladimirov and I. Volovich, "Conservation laws for non-linear equations." Russ. Math. Surv., vol. 40, no. 14, pp. 13-24, 1985, doi: 10.1070/RM1985v040n04ABEH003608.

[16] V. Vladimirov and I. Volovich, "Local and nonlocal currents for nonlinear equation." Theor. Math. Phys., vol. 62, no. 1, pp. 1-20, 1985, doi: 10.1007/BF01034820.

Authors' addresses

\section{Anatolij M. Samoilenko}

The Institute of Mathematics of NAS of Ukraine, and the Department of Applied Mathematics at the University of Agriculture in Krakow, Poland

\section{Yarema A. Prykarpatskyy}

The Institute of Mathematics of NAS of Ukraine, and the Department of Applied Mathematics at the University of Agriculture in Krakow, Poland

E-mail address: yarpryegmail.com

Denis Blackmore

Department of Mathematical Sciences at the New Jersey Institute of Technology (NJIT), Newark NJ 07102, USA

E-mail address: deblac@m.njit.edu

Anatolij K. Prykarpatski

The Institute of Mathematics at the Cracow University of Technology, Poland

E-mail address: pryk. anat@ua.fm 